TOKYO J. Math.

Vol. 21, No. 1,1998

\title{
A Weak Ordering of Positive Dependence Structure of Stochastic Processes
}

\author{
Jong-Il BAEK, ${ }^{*}$ Jeong-Yeol CHOI ${ }^{* *}$ and Dae-Hee RU ${ }^{* * *}$ \\ Won Kwang University and Chung Nam San Up University \\ (Communicated by K. Katayama)
}

\begin{abstract}
In this paper we introduce a new concept of more weakly quadrant dependence of hitting times of stochastic processes. This concept is weaker than the more positively quadrant dependence and it is closed under some statistical operations of weakly positive quadrant dependence (WPQD) ordering.
\end{abstract}

\section{Introduction.}

Lehmann [12] introduced the concept of positive (negative) dependence together with some other dependence concepts. Since then, a great many works have been studied on the subject and its extensions and numerous multivariate inequalities have been obtained. For references of available results, see Karlin and Rinott [11], Ebrahimi and Ghosh [8] and Sampson [13]. Whereas a number of dependence notions exist for multivariate processes (see Friday [10]), recently, Ebrahimi [7] introduced some new dependence concepts of the hitting times of stochastic processes.

Most of the dependence concepts introduced in the literature are stronger than the positive (negative) dependence. For this reason, Baek [2] introduced some new weakly positive quadrant dependence concepts in terms of the finite-dimensional distributions of the hitting times of the components of a vector process. These concepts not only help us to understand structure of functionals such as hitting times of the given vector process but also have the potential for new and useful inequalities for stochastic processes. Moreover, the concept of dependence is a form of qualitative bivariate dependence which has led to many applications in applied probability, reliability, and statistical inference such as analysis of variance, multivariate tests of hypothesis, sequential testing. Like this, since WPQD is a qualitative form of dependence, it would seem difficult, or impossible to compare different pairs of stochastic processes as to their "degree of WPQD-ness". For these reasons, in this paper we introduce a new notion of weakly

Received June 24, 1996

Revised May 21, 1997

** This paper was partially supported by research fund of Won Kwang University, 1997. 
more positive quadrant dependent of two stochastic processes. The importance of this paper lies in the fact that this new notion is weaker than the more positive quadrant dependence. In particular, we give a partial ordering which permits us to compare pairs of WPQD bivariate vector processes of interest as to their "degree of WPQD-ness".

In Section 2, we develop some definitions and notations of WPQD ordering processes. In Section 3, we define a WPQD family of bivariate distributions $H$ which is increasing in $\lambda$ and give some interesting examples of such families.

In Section 4, we derive useful closure properties of WPQD ordering. We show that WPQD ordering is closed under convolution, limit in distribution, compound distribution, mixture of certain types, transformations of univariate increasing functions, and convex combination.

\section{Notation and definitions.}

First, in this section, we present notations and basic facts used throughout the paper. In what follows 'increasing' means non-decreasing and 'positive' means nonnegative.

Suppose that we are given two bivariate stochastic processes $\left\{\left(X_{11}(t), X_{21}(t) \mid t \geq 0\right\}\right.$, $\left\{\left(X_{12}(t), X_{22}(t)\right) \mid t \geq 0\right\}$. The state space of $\left(X_{11}(t), X_{21}(t)\right)$ and $\left(X_{12}(t), X_{22}(t)\right)$ will be taken to be any subset, $E=E_{1} \times E_{2}$, of the plane $\mathbf{R}^{2}$.

For any states $a_{i} \in E_{i}, i=1,2$, we define the random times as follows:

$$
T_{i j}\left(a_{i}\right)=\inf \left\{t \mid X_{i j}(t) \geq a_{i}, 0 \leq t \leq \infty\right\}, \quad j=1,2 .
$$

In other words, $T_{i j}\left(a_{i}\right)$ is the hitting time that the $i j$ th component process $X_{i j}(t)$ reaches or goes above $a_{i}$ (see [7]). If we base the dependence between processes on the dependence of their hitting times, we then have the following definitions.

Definition 2.1 [3]. The bivariate stochastic process $\left\{\left(X_{12}(t), X_{22}(t)\right) \mid t \geq 0\right\}$ is said to be more positively quadrant dependent than $\left\{\left(X_{11}(t), X_{21}(t)\right) \mid t \geq 0\right\}$ if

$$
P\left(T_{12}\left(a_{1}\right)>t_{1}, T_{22}\left(a_{2}\right)>t_{2}\right) \geq P\left(T_{11}\left(a_{1}\right)>t_{1}, T_{21}\left(a_{2}\right)>t_{2}\right)
$$

for all $t_{i} \geq 0, a_{i} \in E_{i}, i=1,2$.

Definition 2.2 [2]. The bivariate stochastic process $\left\{\left(X_{12}(t), X_{22}(t)\right) \mid t \geq 0\right\}$ is said to be weakly positive quadrant dependent of the first type (WPQD1) if

$$
\int_{x_{1}}^{\infty} \int_{x_{2}}^{\infty} P\left(\bigcap_{i=1}^{2}\left(T_{i 2}\left(a_{i}\right)>t_{i}\right)\right) d t_{2} d t_{1} \geq \int_{x_{1}}^{\infty} \int_{x_{2}}^{\infty} \prod_{i=1}^{2} P\left(T_{i 2}\left(a_{i}\right)>t_{i}\right) d t_{2} d t_{1}
$$

for all $x_{i}>0, a_{i} \in E_{i}, i=1,2$.

Definition 2.3 [2]. The bivariate stochastic process $\left\{\left(X_{12}(t), X_{22}(t)\right) \mid t \geq 0\right\}$ is said to be weakly positive quadrant dependent of the second type (WPQD2) if 


$$
\int_{0}^{x_{1}} \int_{0}^{x_{2}} P\left(\bigcap_{i=1}^{2}\left(T_{i 2}\left(a_{i}\right)>t_{i}\right)\right) d t_{2} d t_{1} \geq \int_{0}^{x_{1}} \int_{0}^{x_{2}} \prod_{i=1}^{2} P\left(T_{i 2}\left(a_{i}\right)>t_{i}\right) d t_{2} d t_{1}
$$

for all $x_{i}>0, a_{i} \in E_{i}, i=1,2$.

Moreover, $\left\{\left(X_{12}(t), X_{22}(t)\right) \mid t \geq 0\right\}$ (or the distribution $H$ ) is said to be weakly positive quadrant dependent (WPQD) if they satisfy both WPQD1 and WPQD2.

Definition 2.4 [4]. The bivariate stochastic process $\left\{\left(X_{12}(t), X_{22}(t)\right) \mid t \geq 0\right\}$ is said to be associated if $\operatorname{Cov}\left(f\left(T_{12}\left(a_{1}\right)\right), g\left(T_{22}\left(a_{2}\right)\right)\right) \geq 0$ for all positive increasing convex functions $f$ and $g$ for which the covariance exists and $a_{i} \in E_{i}, i=1,2$.

Definition 2.5 [4]. A stochastic process $\left\{X_{22}(t) \mid t \geq 0\right\}$ is stochastically increasing (SI) in $\left\{X_{12}(t) \mid t \geq 0\right\}$ if $E\left(f\left(T_{22}\left(a_{2}\right)\right) \mid T_{12}\left(a_{1}\right)=t_{1}\right)$ is increasing in $t_{1}$ for all $a_{i} \in E_{i}, i=1,2$, and positive increasing convex function $f$.

Before we state more definitions, we let $\beta=\beta(F, G)$ denote the class of bivariate distribution function $H$ having specified marginal distribution functions $F$ and $G$, where $F$ and $G$ are nondegenerate, and we then consider $\beta^{+}$, a subclass of $\beta$, defined by

$$
\beta^{+}=\left\{H\left(t_{1}, t_{2}\right) \mid H \text { is WPQD, } H\left(t_{1}, \infty\right)=F\left(t_{1}\right), H\left(\infty, t_{2}\right)=G\left(t_{2}\right)\right\} .
$$

Let $H_{1}, H_{2}$ belong to $\beta^{+}$, and use the notations $\bar{H}_{1}=P\left(T_{11}\left(a_{1}\right)>t_{1}, T_{21}\left(a_{2}\right)>t_{2}\right)$, $\bar{H}_{2}=P\left(T_{12}\left(a_{1}\right)>t_{1}, T_{22}\left(a_{2}\right)>t_{2}\right)$.

DEFINITION 2.6. The bivariate distribution $\mathrm{H}_{2}$ is said to be more weakly positive quadrant dependent of the first type than $H_{1}$ if

$$
\int_{x_{1}}^{\infty} \int_{x_{2}}^{\infty} \overline{H_{2}}\left(t_{1}, t_{2}\right) d t_{1} d t_{2} \geq \int_{x_{1}}^{\infty} \int_{x_{2}}^{\infty} \overline{H_{1}}\left(t_{1}, t_{2}\right) d t_{1} d t_{2} \quad \text { for all } x_{i}>0, \quad i=1,2
$$

We write $H_{2}>\left(\right.$ WPQD1) $H_{1}$.

Definition 2.7. The bivariate distribution $\mathrm{H}_{2}$ is said to be more weakly positive quadrant dependent of the second type than $H_{1}$ if

$$
\int_{0}^{x_{1}} \int_{0}^{x_{2}} \overline{H_{2}}\left(t_{1}, t_{2}\right) d t_{1} d t_{2} \geq \int_{0}^{x_{1}} \int_{0}^{x_{2}} \overline{H_{1}}\left(t_{1}, t_{2}\right) d t_{1} d t_{2} \text { for all } x_{i}>0, \quad i=1,2 \text {. }
$$

We write $H_{2}>$ (WPQD2) $H_{1}$.

Moreover, the bivariate distribution $H_{2}$ is said to be more weakly positive quadrant dependent than $H_{1}$ if they satisfy both $H_{2}>$ (WPQD1) $H_{1}$ and $H_{2}>$ (WPQD2) $H_{1}$. We write $H_{2}>$ (WPQD) $H_{1}$.

REMARK 1. (a) An equivalent form of (2.1) is 


$$
\int_{x_{1}}^{\infty} \int_{x_{2}}^{\infty} H_{2}\left(t_{1}, t_{2}\right) d t_{1} d t_{2} \geq \int_{x_{1}}^{\infty} \int_{x_{2}}^{\infty} H_{1}\left(t_{1}, t_{2}\right) d t_{1} d t_{2} \text { for all } x_{i}>0, \quad i=1,2 .
$$

(b) An equivalent form of (2.2) is

$$
\int_{0}^{x_{1}} \int_{0}^{x_{2}} H_{2}\left(t_{1}, t_{2}\right) d t_{1} d t_{2} \geq \int_{0}^{x_{1}} \int_{0}^{x_{2}} H_{1}\left(t_{1}, t_{2}\right) d t_{1} d t_{2} \text { for all } x_{i}>0, \quad i=1,2 \text {. }
$$

From the Definitions 2.1, 2.6, 2.7 and [1] we then have the following theorem.

THEOREM 2.8. Let $\mathrm{H}_{1}$ and $\mathrm{H}_{2}$ be bivariate distribution with specified marginals $F$ and $G$. Assume that the bivariate distribution $\mathrm{H}_{2}$ is more positively quadrant dependent than $H_{1}$. Then $H_{2}$ is more weakly positive quadrant dependent than $H_{1}$.

\section{WPQD increasing in a parameter.}

Before we state the definiton, we let $t_{1} \wedge t_{2}=\min \left\{t_{1}, t_{2}\right\}$. Within $\beta^{+}$there are cumulative distribution function $H_{0}$ and $H^{*}$ defined by

$$
H_{0}\left(t_{1}, t_{2}\right)=F\left(t_{1}\right) \cdot G\left(t_{2}\right), \quad H^{*}\left(t_{1}, t_{2}\right)=F\left(t_{1}\right) \wedge G\left(t_{2}\right)
$$

such that for any $H \in \beta$,

$$
H_{0}\left(t_{1}, t_{2}\right) \leq H\left(t_{1}, t_{2}\right) \leq H^{*}\left(t_{1}, t_{2}\right) \text { for all } t_{i} \geq 0, \quad i=1,2 .
$$

In this section, we define WPQD families increasing in a parameter and give some interesting examples of such families.

Definition 3.1. A family of WPQD distributions $H=\left\{H_{\lambda}\left(t_{1}, t_{2}\right) \mid \lambda \in \Lambda \subset \mathbf{R}\right\}$ is said to be increasing weakly positive quadrant dependent in $\lambda$ if for $\lambda, \lambda^{\prime} \in \Lambda$,

$$
\lambda^{\prime}>\lambda \rightarrow H_{\lambda^{\prime}}>\text { (WPQD) } H_{\lambda} .
$$

Next we present some examples of families which are increasing weakly positive quadrant dependent in the indexing parameter.

EXAMPLE 3.2. Consider the bivariate distribution

$$
H_{\alpha}\left(t_{1}, t_{2}\right)=F\left(t_{1}\right) G\left(t_{2}\right)\left\{1+\alpha \bar{F}\left(t_{1}\right) \bar{G}\left(t_{2}\right)\right\}, \quad 0 \leq \alpha<1 .
$$

Then for $0 \leq \alpha_{1}<\alpha_{2}<1$,

$$
\begin{aligned}
& \int_{x_{1}}^{\infty} \int_{x_{2}}^{\infty} \bar{H}_{\alpha_{2}}\left(t_{1}, t_{2}\right) d t_{1} d t_{2} \geq \int_{x_{1}}^{\infty} \int_{x_{2}}^{\infty} \bar{H}_{\alpha_{1}}\left(t_{1}, t_{2}\right) d t_{1} d t_{2}, \\
& \int_{0}^{x_{1}} \int_{0}^{x_{2}} \bar{H}_{\alpha_{2}}\left(t_{1}, t_{2}\right) d t_{1} d t_{2} \geq \int_{0}^{x_{1}} \int_{0}^{x_{2}} \bar{H}_{\alpha_{1}}\left(t_{1}, t_{2}\right) d t_{1} d t_{2} .
\end{aligned}
$$

Hence $H_{\alpha}\left(t_{1}, t_{2}\right)$ is increasing WPQD in $\alpha$. 
EXAMPLE 3.3. Let $\beta_{c}^{+}$be the class of all convex combinations

$$
\left\{H\left(t_{1}, t_{2}\right) \mid H=(1-\alpha) H_{0}+\alpha H^{*}, 0 \leq \alpha \leq 1\right\} \text {. }
$$

First of all, we will show that $\beta_{c}^{+}$is a subclass of $\beta^{+}$. Suppose $H=(1-\alpha) H_{0}+\alpha H^{*}$. If $\alpha=0$ or $\alpha=1$, then $H \in \beta^{+}$. For $0<\alpha<1$,

$$
\begin{aligned}
\lim _{t_{1} \rightarrow \infty} H\left(t_{1}, t_{2}\right) & =\lim _{t_{1} \rightarrow \infty}\left[(1-\alpha) F\left(t_{1}\right) G\left(t_{2}\right)+\alpha\left(F\left(t_{1}\right) \wedge G\left(t_{2}\right)\right)\right] \\
& =(1-\alpha) G\left(t_{2}\right)+\alpha G\left(t_{2}\right)=G\left(t_{2}\right), \\
\lim _{t_{2} \rightarrow \infty} H\left(t_{1}, t_{2}\right) & =\lim _{t_{2} \rightarrow \infty}\left[(1-\alpha) F\left(t_{1}\right) G\left(t_{2}\right)+\alpha\left(F\left(t_{1}\right) \wedge G\left(t_{2}\right)\right)\right] \\
& =(1-\alpha) F\left(t_{1}\right)+\alpha F\left(t_{1}\right)=F\left(t_{1}\right) .
\end{aligned}
$$

It is clear that $H$ has marginals $F$ and $G$. Moreover,

$$
\begin{aligned}
\int_{x_{1}}^{\infty} \int_{x_{2}}^{\infty} H\left(t_{1}, t_{2}\right) d t_{1} d t_{2}= & \int_{x_{1}}^{\infty} \int_{x_{2}}^{\infty}\left[(1-\alpha) F\left(t_{1}\right) G\left(t_{2}\right)+\alpha\left(F\left(t_{1}\right) \wedge G\left(t_{2}\right)\right)\right] d t_{1} d t_{2} \\
& \left\{\begin{array}{l}
\int_{x_{1}}^{\infty} \int_{x_{2}}^{\infty}\left[(1-\alpha) F\left(t_{1}\right) G\left(t_{2}\right)+\alpha F\left(t_{1}\right)\right] d t_{1} d t_{2} \text { if } t_{2} \geq t_{1} \\
=\int_{x_{1}}^{\infty} \int_{x_{2}}^{\infty} F\left(t_{1}\right) G\left(t_{2}\right)\left[1+\alpha \frac{\bar{G}\left(t_{2}\right)}{G\left(t_{2}\right)}\right] d t_{1} d t_{2}, \\
\int_{x_{1}}^{\infty} \int_{x_{2}}^{\infty}\left[(1-\alpha) F\left(t_{1}\right) G\left(t_{2}\right)+\alpha G\left(t_{2}\right)\right] d t_{1} d t_{2} \text { if } t_{2}<t_{1}
\end{array}\right. \\
\geq & \int_{x_{1}}^{\infty} \int_{x_{2}}^{\infty} F\left(t_{1}\right) G\left(t_{2}\right) d t_{1} d t_{2} .
\end{aligned}
$$

Hence $H$ is WPQD1. The proof of the WPQD2 is similar to the proof of the WPQD1. Therefore $H \in \beta^{+}$. Thus $\beta_{c}^{+} \subset \beta^{+}$.

For $0 \leq \alpha_{1} \leq \alpha_{2}<1$,

$$
\begin{aligned}
& \int_{x_{1}}^{\infty} \int_{x_{2}}^{\infty}\left[\left(1-\alpha_{2}\right) F\left(t_{1}\right) G\left(t_{2}\right)+\alpha_{2}\left(F\left(t_{1}\right) \wedge G\left(t_{2}\right)\right)\right] d t_{1} d t_{2} \\
& \quad-\int_{x_{1}}^{\infty} \int_{x_{2}}^{\infty}\left[\left(1-\alpha_{1}\right) F\left(t_{1}\right) G\left(t_{2}\right)+\alpha_{1}\left(F\left(t_{1}\right) \wedge G\left(t_{2}\right)\right)\right] d t_{1} d t_{2} \geq 0, \\
& \int_{0}^{x_{1}} \int_{0}^{x_{2}}\left[\left(1-\alpha_{2}\right) F\left(t_{1}\right) G\left(t_{2}\right)+\alpha_{2}\left(F\left(t_{1}\right) \wedge G\left(t_{2}\right)\right)\right] d t_{1} d t_{2} \\
& -\int_{0}^{x_{1}} \int_{0}^{x_{2}}\left[\left(1-\alpha_{1}\right) F\left(t_{1}\right) G\left(t_{2}\right)+\alpha_{1}\left(F\left(t_{1}\right) \wedge G\left(t_{2}\right)\right)\right] d t_{1} d t_{2} \geq 0 .
\end{aligned}
$$


Hence $\beta_{c}^{+}$is increasing WPQD in $\alpha$.

\section{Closure properties of $\left(\beta^{+},>(\mathrm{WPQD})\right)$.}

In this section, we establish preservation of the WPQD ordering under convolution, limit in distribution, compound distribution, mixture of a certain type, transformations of univariate increasing convex functions, and convex combination. First note that by Theorem 3 of Alzaid, (2.1) and (2.2) are equivalent to $E\left(f\left(T_{12}\left(a_{1}\right)\right) g\left(T_{22}\left(a_{2}\right)\right)\right) \geq$ $E\left(f\left(T_{11}\left(a_{1}\right)\right) g\left(T_{21}\left(a_{2}\right)\right)\right)$ for all increasing positive convex functions $f$ and $g$.

Below, we show that the ordering is preserved under convolution. We need the following lemma which is of independent interest.

LEMMA 4.1. Let (a) $\left\{\left(X_{11}(t), X_{21}(t)\right) \mid t \geq 0\right\}$ and $\left\{\left(X_{12}(t), X_{22}(t)\right) \mid t \geq 0\right\}$ have distributions $H_{1}$ and $H_{2}$, where $H_{1}, H_{2}$ belong to $\beta^{+}$, (b) $\left\{\left(X_{12}(t), X_{22}(t)\right) \mid t \geq 0\right\}>$ (WPQD) $\left\{\left(X_{11}(t), X_{21}(t)\right) \mid t \geq 0\right\}$, and (c) $\left(Z_{1}, Z_{2}\right)$ with an arbitrary WPQD distribution function $H$ independent of both of $\left\{\left(X_{11}(t), X_{21}(t)\right) \mid t \geq 0\right\}$ and $\left\{\left(X_{12}(t), X_{22}(t)\right) \mid t \geq 0\right\}$. Then $\left(X_{12}(t)+\right.$ $\left.Z_{1}, X_{22}(t)+Z_{2}\right)>$ (WPQD) $\left(X_{11}(t)+Z_{1}, X_{21}(t)+Z_{2}\right)$.

Proof. First, we will show that $\left(X_{12}(t)+Z_{1}, X_{22}(t)+Z_{2}\right)$ is WPQD. Consider any hitting times $W_{i j}\left(a_{i}\right)$ given by $W_{i j}\left(a_{i}\right)=\inf \left\{t \mid X_{i j}(t)+Z_{i} \geq a_{i}, t \geq 0\right\}, i, j=1,2$. Then,

$$
\begin{aligned}
\operatorname{Cov}( & \left.f\left(W_{12}\left(a_{1}\right)\right), g\left(W_{22}\left(a_{2}\right)\right)\right)=\operatorname{Cov}\left(f\left(T_{12}\left(a_{1}-Z_{1}\right)\right), g\left(T_{22}\left(a_{2}-Z_{2}\right)\right)\right) \\
= & \operatorname{Cov}\left(E\left(f\left(T_{12}\left(a_{1}-Z_{1}\right)\right) \mid Z_{1}, Z_{2}\right), E\left(g\left(T_{22}\left(a_{2}-Z_{2}\right)\right) \mid Z_{1}, Z_{2}\right)\right) \\
& +E\left(\operatorname{Cov}\left(f\left(T_{12}\left(a_{1}-Z_{1}\right)\right), g\left(T_{22}\left(a_{2}-Z_{2}\right)\right) \mid Z_{1}, Z_{2}\right)\right) \geq 0 .
\end{aligned}
$$

Note that the first and second terms are greater than or equal to zero for any increasing convex functions $f$ and $g$. Thus by Theorem 3 of Alzaid (1990), $\left(X_{12}(t)+Z_{1}, X_{22}(t)+Z_{2}\right)$ is WPQD. Similarly we can show that $\left(X_{11}(t)+Z_{1}, X_{21}(t)+Z_{2}\right)$ is also WPQD.

Next, we will show that $\left(X_{12}(t)+Z_{1}, X_{22}(t)+Z_{2}\right)>\left(\right.$ WPQD) $\left(X_{11}(t)+Z_{1}, X_{21}(t)+\right.$ $\left.Z_{2}\right)$, i.e.,

$$
E\left(f\left(T_{12}\left(a_{1}-Z_{1}\right)\right) g\left(T_{22}\left(a_{2}-Z_{2}\right)\right)\right) \geq E\left(f\left(T_{11}\left(a_{1}-Z_{1}\right)\right) g\left(T_{21}\left(a_{2}-Z_{2}\right)\right)\right)
$$

for any increasing convex functions $f$ and $g$. Now,

$$
\begin{aligned}
& E\left(f\left(T_{12}\left(a_{1}-Z_{1}\right)\right) g\left(T_{22}\left(a_{2}-Z_{2}\right)\right)\right)=E\left(E\left(f\left(T_{12}\left(a_{1}-Z_{1}\right)\right) g\left(T_{22}\left(a_{2}-Z_{2}\right)\right) \mid Z_{1}, Z_{2}\right)\right) \\
& \quad=E\left(E\left(f\left(T_{12}\left(a_{1}-Z_{1}\right)\right) g\left(T_{22}\left(a_{2}-Z_{2}\right)\right)\right)\right) \geq E\left(E\left(f\left(T_{11}\left(a_{1}-Z_{1}\right)\right) g\left(T_{21}\left(a_{2}-Z_{2}\right)\right)\right)\right) \\
& \quad=E\left(f\left(T_{11}\left(a_{1}-Z_{1}\right)\right) g\left(T_{21}\left(a_{2}-Z_{2}\right)\right)\right) .
\end{aligned}
$$

The inequality follows from the assumption that $\left(X_{12}(t), X_{22}(t)\right)>(\operatorname{WPQD})\left(X_{11}(t)\right.$, $\left.X_{21}(t)\right)$.

THEOREM 4.2. Suppose that the stochastic process (a) $\left\{\left(X_{12}(t), X_{22}(t)\right) \mid t \geq 0\right\}$ is more weakly positive quadrant dependent than $\left\{\left(X_{11}(t), X_{21}(t)\right) \mid t \geq 0\right\},(\mathrm{b})\left\{\left(Y_{12}(t), Y_{22}(t)\right) \mid t \geq 0\right\}$ 
is more weakly positive quadrant dependent than $\left\{\left(Y_{11}(t), Y_{21}(t)\right) \mid t \geq 0\right\}$, and $(\mathrm{c})$ let $\left\{\left(X_{12}(t)\right.\right.$, $\left.\left.X_{22}(t)\right) \mid t \geq 0\right\}$ and $\left\{\left(Y_{12}(t), Y_{22}(t)\right) \mid t \geq 0\right\}$ be independent processes, $\left\{\left(X_{11}(t), X_{21}(t) \mid t \geq 0\right\}\right.$ and $\left\{\left(Y_{11}(t), Y_{21}(t)\right) \mid t \geq 0\right\}$ be independent processes. Then $\left\{\left(X_{12}(t)+Y_{12}(t), X_{22}(t)+\right.\right.$ $\left.\left.Y_{22}(t)\right) \mid t \geq 0\right\}>(\mathrm{WPQD})\left\{\left(X_{11}(t)+Y_{11}(t), X_{21}(t)+Y_{21}(t)\right) \mid t \geq 0\right\}$.

Proof. By assumption, $\left(X_{12}(t), X_{22}(t)\right)>$ (WPQD) $\left(X_{11}(t), X_{21}(t)\right)$. Specifying $\left(Z_{1}(t), Z_{2}(t)\right)$ to be $\left(Y_{12}(t), Y_{22}(t)\right)$, we apply Lemma 4.1 to obtain

$$
\left(X_{12}(t)+Y_{12}(t), X_{22}(t)+Y_{22}(t)\right)>(\mathrm{WPQD})\left(X_{11}(t)+Y_{12}(t), X_{21}(t)+Y_{22}(t)\right) .
$$

Next, we use the assumption $\left(Y_{12}(t), Y_{22}(t)\right)>$ (WPQD) $\left(Y_{11}(t), Y_{21}(t)\right)$, specifying $\left(Z_{1}(t), Z_{2}(t)\right)$ to be $\left(X_{11}(t), X_{21}(t)\right)$, and again use Lemma 4.1 yielding

$$
\left(X_{11}(t)+Y_{12}(t), X_{21}(t)+Y_{22}(t)\right)>(\mathrm{WPQD})\left(X_{11}(t)+Y_{11}(t), X_{21}(t)+Y_{21}(t)\right) .
$$

By combining (4.1) and (4.2),

$$
\begin{aligned}
\left(X_{12}(t)+Y_{12}(t), X_{22}(t)+Y_{22}(t)\right) & >(\mathrm{WPQD})\left(X_{11}(t)+Y_{12}(t), X_{21}(t)+Y_{22}(t)\right) \\
& >(\mathrm{WPQD})\left(X_{11}(t)+Y_{11}(t), X_{21}(t)+Y_{21}(t)\right) .
\end{aligned}
$$

Thus

$$
\left(X_{12}(t)+Y_{12}(t), X_{22}(t)+Y_{22}(t)\right)>(\mathrm{WPQD})\left(X_{11}(t)+Y_{11}(t), X_{21}(t)+Y_{21}(t)\right) .
$$

This completes the proof.

The next theorem demonstrates that, under suitable conditions, limits of the WPQD ordering processes inherit the WPQD ordering structure.

THEOREM 4.3. Let (a) $\left\{\left(X_{n 1}(t), X_{n 2}(t)\right) \mid t \geq 0\right\},\left\{\left(Y_{n 1}(t), Y_{n 2}(t)\right) \mid t \geq 0\right\}$ have distributions $H_{n}, H_{n}^{\prime}$ for every $n$ and $H_{n}>$ (WPQD) $H_{n}^{\prime}$, (b) $\left\{\left(X_{1}(t), X_{2}(t)\right) \mid t \geq 0\right\},\left\{\left(Y_{1}(t)\right.\right.$, $\left.\left.Y_{2}(t)\right) \mid t \geq 0\right\}$ have distributions $H, H^{\prime}$, (c) $\left\{\left(X_{n 1}(t), X_{n 2}(t)\right) \mid t \geq 0\right\},\left\{\left(Y_{n 1}(t), Y_{n 2}(t)\right) \mid t \geq 0\right\}$, $\left\{\left(X_{1}(t), X_{2}(t)\right) \mid t \geq 0\right\}$ and $\left\{\left(Y_{1}(t), Y_{2}(t)\right) \mid t \geq 0\right\}$ have all sample paths and they are right continuous on $[0, \infty)$ with finite left limits at all $t$, and (d) $H_{n} \stackrel{w}{\longrightarrow} H$ and $H_{n}^{\prime} \stackrel{w}{\longrightarrow} H^{\prime}$ as $n \rightarrow \infty$, respectively. Then $H>$ (WPQD) $H^{\prime}$.

Proof. Denote by $C(H)$ and $C\left(H^{\prime}\right)$ the sets of continuity points of $H$ and $H^{\prime}$, respectively. Let $D=C(H) \cap C\left(H^{\prime}\right)$. It follows from our assumptions that $H\left(t_{1}, t_{2}\right) \geq$ $H^{\prime}\left(t_{1}, t_{2}\right)$ for all $\left(t_{1}, t_{2}\right) \in D$. Since $D$ is a dense set in $\mathbf{R}^{2}, H>$ (WPQD) $H^{\prime}$.

The following theorem is another application of Theorem 4.2 which is very important in recognizing WPQD ordering in compound distributions which arise naturally in stochastic processes.

THEOREM 4.4. Let (a) $\left(Y_{1}, S_{1}\right),\left(Y_{2}, S_{2}\right), \cdots$ be independent random processes, (b) $\left(X_{1}, K_{1}\right),\left(X_{2}, K_{2}\right), \cdots$ be independent random processes, (c) $\left(Y_{i}, S_{i}\right)$ and $\left(X_{i}, K_{i}\right), i=$ $1,2, \cdots, n$, are WPQD random process $(\mathrm{d})\left(Y_{i}, S_{i}\right)>$ (WPQD) $\left(X_{i}, K_{i}\right), i=1,2, \cdots$, and (e) $N(t)$ be a Poisson process which is independent of $\left(Y_{i}, S_{i}\right)$ and $\left(X_{i}, K_{i}\right), i=1,2, \cdots$. 
Then

$$
\left(Z_{12}(t)=\sum_{i=1}^{N(t)} Y_{i}, Z_{22}(t)=\sum_{i=1}^{N(t)} S_{i}\right)>(\mathrm{WPQD})\left(Z_{11}(t)=\sum_{i=1}^{N(t)} X_{i}, Z_{21}(t)=\sum_{i=1}^{N(t)} K_{i}\right)
$$

Proof. This can be proved by the similar method as Theorem 4 in [7].

Our next result deals with the preservation of the WPQD ordering under mixture. In order to motivate our definition of a subclass of $\beta^{+}$in which the WPQD ordering is preserved under mixture we need Proposition 4.5.

Proposition 4.5. Let (a) $\left\{\left(X_{12}(t), X_{22}(t)\right) \mid t \geq 0\right\}$ given a scalar $\lambda$, a stochastic process be conditionally WPQD, and (b) $\left\{X_{i 2}(t) \mid t \geq 0\right\}$ be $S I$ in $\lambda$, for each $i=1,2$, (c) $\lambda$ be associated. Then $\left\{\left(X_{12}(t), X_{22}(t)\right) \mid t \geq 0\right\}$ is WPQD.

Proof. Let $f$ and $g$ be increasing positive convex functions for which the covariance exists. Then

$$
\begin{aligned}
& \operatorname{Cov}\left(f\left(T_{12}\left(a_{1}\right)\right), g\left(T_{22}\left(a_{2}\right)\right)\right) \\
& \quad=E\left[\operatorname{Cov}\left(f\left(T_{12}\left(a_{1}\right)\right), g\left(T_{22}\left(a_{2}\right)\right) \mid \lambda\right)\right]+\operatorname{Cov}\left[E\left(f\left(T_{12}\left(a_{1}\right)\right) \mid \lambda\right), E\left(g\left(T_{22}\left(a_{2}\right)\right) \mid \lambda\right)\right] .
\end{aligned}
$$

Conditioned on $\lambda,\left(X_{12}(t), X_{22}(t)\right)$ is WPQD1 (WPQD2). Thus by Theorem 3 of Alzaid (1990) the first term on the right hand side of (4.3) is positive. From Definition 2.5 the conditional expectations in the second term on the right hand side of (4.3) are increasing functions of $\lambda$. Since $\lambda$ is associated, the covariance of the conditional expectations in the second term is positive. It follows that $\operatorname{Cov}\left(f\left(T_{12}\left(a_{1}\right)\right), g\left(T_{22}\left(a_{2}\right)\right)\right) \geq 0$. Thus $\left\{\left(X_{12}(t), X_{22}(t)\right) \mid t \geq 0\right\}$ is WPQD.

We may now define the class $\beta_{\lambda}^{+}$by

$$
\begin{aligned}
\beta_{\lambda}^{+}=\left\{H_{\lambda} \mid\right. & H\left(t_{1}, \infty \mid \lambda\right)=F\left(t_{1} \mid \lambda\right), H\left(\infty, t_{2} \mid \lambda\right)=G\left(t_{2} \mid \lambda\right), \\
& \left.H_{\lambda} \mid \lambda \text { is WPQD, and both } F \text { and } G \text { are SI in } \lambda\right\} .
\end{aligned}
$$

Now consider $\left(\beta_{\lambda}^{+},>(\mathrm{WPQD})\right)$. The following theorem shows that if two elements of $\beta_{\lambda}^{+}$are ordered according to $>$(WPQD), then after mixing $\lambda$, the resulting element in $\beta^{+}$preserves the same order.

THEOREM 4.6. Let $\left(X_{12}(t), X_{22}(t)\right) \mid \lambda$ and $\left(X_{11}(t), X_{21}(t)\right) \mid \lambda$ belong to $\beta_{\lambda}^{+}$and $\left(\left(X_{12}(t), X_{22}(t)\right) \mid \lambda\right)>(\mathrm{WPQD})\left(\left(X_{11}(t), X_{21}(t)\right) \mid \lambda\right)$ for all $\lambda$. Then, unconditionally, $\left(X_{12}(t), X_{22}(t)\right),\left(X_{11}(t), X_{21}(t)\right)$ belong to $\beta^{+}$and $\left(X_{12}(t), X_{22}(t)\right)>$ (WPQD) $\left(X_{11}(t), X_{21}(t)\right)$.

Proof. From Proposition 4.5, $\left(X_{12}(t), X_{22}(t)\right)$ and $\left(X_{11}(t), X_{21}(t)\right)$ are WPQD. Let $f$ and $g$ be positive increasing convex functions. Then

$$
\begin{aligned}
& E\left(f\left(T_{12}\left(a_{1}\right)\right) g\left(T_{22}\left(a_{2}\right)\right)\right)=E_{\lambda}\left(E\left(f\left(T_{12}\left(a_{1}\right)\right) g\left(T_{22}\left(a_{2}\right)\right) \mid \lambda\right)\right) \\
& \quad \geq E_{\lambda}\left(E\left(f\left(T_{11}\left(a_{1}\right)\right) g\left(T_{21}\left(a_{2}\right)\right) \mid \lambda\right)\right)=E\left(f\left(T_{11}\left(a_{1}\right)\right) g\left(T_{21}\left(a_{2}\right)\right)\right) .
\end{aligned}
$$


The inequality comes from the fact that $\left(X_{12}(t), X_{22}(t)\right)\left|\lambda>(\mathrm{WPQD})\left(X_{11}(t), X_{21}(t)\right)\right| \lambda$ for all $\lambda$

Next, we show that the WPQD ordering is invariant under transformations of stochastic processes by increasing convex functions.

RESUlt 4.7. Let (a) $\left\{\left(X_{i j}(t), X_{i j}^{\prime}(t)\right)^{H_{j}} \mid t \geq 0\right\}, i=1,2,3, \cdots, n$, be n-independent pairs from a bivariate distribution $H_{j}$ with continuous increasing sample paths, $j=1,2$, (b) $H_{1}$ and $H_{2}$ belong to $\beta^{+}$such that $H_{2}>$ (WPQD) $H_{1}$, and (c) $f_{1}$ and $f_{2}$ are positive convex functions and they are increasing in each of their arguments when all other arguments are fixed. Then $\left(Y_{12}(t), Y_{22}(t)\right)>(\mathrm{WPQD})\left(Y_{11}(t), Y_{21}(t)\right)$, where the processes $Y_{i j}(t)$ are given by $Y_{1 i}(t)=f_{1}\left(X_{1 i}(t), \cdots, X_{n i}(t)\right), Y_{2 i}(t)=f_{2}\left(X_{1 i}^{\prime}(t), \cdots, X_{n i}^{\prime}(t)\right), i=1,2$.

Proof. First, we will show that the WPQD1 ordering holds. The proof will be given for the case $n=2$. For the general $n$, the proof is similar.

Fix $t_{i} \geq 0, i=1,2$, and introduce the variables $V_{i}=X_{2 i}\left(t_{i}\right), V_{i}^{\prime}=X_{2 i}^{\prime}\left(t_{i}\right), U_{i}=$ $\sup _{0 \leq s<t_{i}}\left(f_{1}\left(X_{1 i}(s), X_{2 i}(s)\right)\right)$, and $U_{i}^{\prime}=\sup _{0 \leq s<t_{i}}\left(f_{2}\left(X_{1 i}^{\prime}(s), X_{2 i}^{\prime}(s)\right)\right), i=1,2$, where for simplicity, $t_{1}, t_{2}$ have been suppressed in $V_{i}, V_{i}^{\prime}, U_{i}$ and $U_{i}^{\prime}$. Consider any hitting times of $Y_{1 i}(s)=f_{1}\left(X_{1 i}(s), X_{2 i}(s)\right), Y_{2 i}(s)=f_{2}\left(X_{1 i}^{\prime}(s), X_{2 i}^{\prime}(s)\right), i=1,2$, given by

$$
W_{i j}\left(a_{i}\right)=\inf \left\{s \mid Y_{i j}(s) \geq a_{i}, s \geq 0\right\}, \quad i, j=1,2 .
$$

It suffices to show that

$$
\begin{aligned}
\int_{x_{1}}^{\infty} & \int_{x_{2}}^{\infty} P\left(W_{12}\left(a_{1}\right)>t_{1}, W_{22}\left(a_{2}\right)>t_{2}\right) d t_{1} d t_{2} \\
& \geq \int_{x_{1}}^{\infty} \int_{x_{2}}^{\infty} P\left(W_{11}\left(a_{1}\right)>t_{1}, W_{21}\left(a_{2}\right)>t_{2}\right) d t_{1} d t_{2}, \quad i=1,2 .
\end{aligned}
$$

Note the facts that $U_{i}=\sup _{0 \leq s<t_{i}}\left(f_{1}\left(X_{1 i}(s), V_{i}\right)\right), U_{i}^{\prime}=\sup _{0 \leq s<t_{i}}\left(f_{2}\left(X_{1 i}^{\prime}(s), V_{i}^{\prime}\right)\right), i=1,2$, and that by hypothesis, $\left(V_{2}, V_{2}^{\prime}\right)>($ WPQD1 $)\left(V_{1}, V_{1}^{\prime}\right)$. Now, we obtain

$$
\begin{aligned}
& \int_{x_{1}}^{\infty} \int_{x_{2}}^{\infty} P\left(W_{12}\left(a_{1}\right)>t_{1}, W_{22}\left(a_{2}\right)>t_{2}\right) d t_{1} d t_{2} \\
& \quad=\int_{x_{1}}^{\infty} \int_{x_{2}}^{\infty} P\left(U_{1}<a_{1}, U_{2}<a_{2}\right) d t_{1} d t_{2} \\
& \quad=\int_{x_{1}}^{\infty} \int_{x_{2}}^{\infty} E\left[P\left(U_{1}<a_{1}, U_{2}<a_{2} \mid V_{2}, V_{2}^{\prime}\right)\right] d t_{1} d t_{2} \\
& \geq \int_{x_{1}}^{\infty} E\left[P\left(U_{1}<a_{1} \mid V_{2}\right)\right] d t_{1} \int_{x_{2}}^{\infty} E\left[P\left(U_{2}<a_{2} \mid V_{2}^{\prime}\right)\right] d t_{2} \\
& \quad \geq \int_{x_{1}}^{\infty} \int_{x_{2}}^{\infty} E\left[P\left(U_{1}<a_{1}, U_{2}<a_{2} \mid V_{1}, V_{1}^{\prime}\right)\right] d t_{1} d t_{2}
\end{aligned}
$$




$$
=\int_{x_{1}}^{\infty} \int_{x_{2}}^{\infty} P\left(W_{11}\left(a_{1}\right)>t_{1}, W_{21}\left(a_{2}\right)>t_{2}\right) d t_{1} d t_{2} .
$$

The proof of the WPQD2 ordering is similar.

We now turn our attention to a simple but important property of the class $\beta^{+}$.

RESULT 4.8. The class $\beta^{+}=\left\{H \mid H\left(t_{1}, t_{2}\right)\right.$ is WPQD, $H\left(t_{1}, \infty\right)=F\left(t_{1}\right), H\left(\infty, t_{2}\right)=$ $\left.G\left(t_{2}\right)\right\}$ is closed under convex combination.

Proof. Let $H_{1}, H_{2} \in \beta^{+}$and for $\alpha \in(0,1), H=\alpha H_{1}+(1-\alpha) H_{2}$. Then we will show that $H$ is a convex combination of $H_{1}$ and $H_{2}$. Since each of $H_{1}$ and $H_{2} \in \beta^{+}$,

$$
\begin{aligned}
\int_{x_{1}}^{\infty} \int_{x_{2}}^{\infty} P_{H}\left(T_{12}\left(a_{1}\right)>t_{1}, T_{22}\left(a_{2}\right)>t_{2}\right) d t_{1} d t_{2} \\
=\alpha \int_{x_{1}}^{\infty} \int_{x_{2}}^{\infty} P_{H_{1}}\left(T_{12}\left(a_{1}\right)>t_{1}, T_{22}\left(a_{2}\right)>t_{2}\right) d t_{1} d t_{2} \\
\quad+(1-\alpha) \int_{x_{1}}^{\infty} \int_{x_{2}}^{\infty} P_{H_{2}}\left(T_{12}\left(a_{1}\right)>t_{1}, T_{22}\left(a_{2}\right)>t_{2}\right) d t_{1} d t_{2} \\
\geq \alpha \int_{x_{1}}^{\infty} \int_{x_{2}}^{\infty} P_{H}\left(T_{12}\left(a_{1}\right)>t_{1}\right) P_{H}\left(T_{22}\left(a_{2}\right)>t_{2}\right) d t_{1} d t_{2} \\
\quad+(1-\alpha) \int_{x_{1}}^{\infty} \int_{x_{2}}^{\infty} P_{H}\left(T_{12}\left(a_{1}\right)>t_{1}\right) P_{H}\left(T_{22}\left(a_{2}\right)>t_{2}\right) d t_{1} d t_{2} \\
=\int_{x_{1}}^{\infty} \int_{x_{2}}^{\infty} P_{H}\left(T_{12}\left(a_{1}\right)>t_{1}\right) P_{H}\left(T_{22}\left(a_{2}\right)>t_{2}\right) d t_{1} d t_{2} .
\end{aligned}
$$

Hence $H$ is WPQD1. The proof of the WPQD2 ordering is similar to the proof of the WPQD1. Moreover,

$$
\begin{aligned}
& \lim _{t_{1} \rightarrow \infty} H\left(t_{1}, t_{2}\right)=\alpha G\left(t_{2}\right)+(1-\alpha) G\left(t_{2}\right)=G\left(t_{2}\right), \\
& \lim _{t_{2} \rightarrow \infty} H\left(t_{1}, t_{2}\right)=\alpha F\left(t_{1}\right)+(1-\alpha) F\left(t_{1}\right)=F\left(t_{1}\right) .
\end{aligned}
$$

It follows from (4.4), (4.5), (4.6) that $H \in \beta^{+}$. Thus $\beta^{+}$is closed under convex combination.

\section{References}

[ 1 ] A. A. Alzaid, A weak quadrant dependence concept with applications, Comm. Statist. Stoch. Models 6 (1990), 353-363.

[2] J. I. BAEK, A weakly dependence concept of bivariate stochastic processes, Comm. Korean Math. Soc. 11 (1996), 831-839. 
[3] J. I. BAEK, A weakly dependence structure of multivariate processes, Statist. Probab. Lett. 34 (1997), 355-363.

[4] R. Barlow and F. Proschan, Statistical Theory of Reliability and Life Testing: Probability Models, Holt, Rinehart and Winston (1975).

[5] R. Bergmann, Some class of semi-ordering relations for random vectors and their use for comparing covariances, Math. Nachr. 82 (1978), 103-114.

[6] N. Ebrahimi, The ordering of negative quadrant dependence, Comm. Statist. Theory Methods 11(12) (1982), 2389-2399.

[ 7 ] N. Ebrahimi, Bivariate processes with positive or negative dependent structures, J. Appl. Probab. 24 (1987), 115-122

[ 8 ] N. Ebrahimi and M. Ghosh, Multivariate negative dependence, Commun. Statist. A 10 (1981), 307337.

[9] N. EbRahimi and T. Ramallingam, On the dependence structure of hitting times of univariate processes, J. Appl. Prob. 25 (1988), 355-362.

[10] D. S. FrIDAY, Dependence concepts for stochastic processes, Proc. Nato Adv. Study Institutes Series 5 (1981), 349-361.

[11] S. KARLIN and Y. RinOtT, Classes of orderings of measures and related correlation inequalities, J. Multivariate Anal. 10 (1980), 467-498.

[12] E. Lehmann, Some concepts of dependence, Ann. Math. Statist. 37 (1966), 1137-1153.

[13] A. R. Sampson, Positive dependence properties of elliptically symmetric distribution, J. Multivariate Anal. 13 (1983), 375-381.

Present Addresses:

JoNG-IL BAEK and JeONG-YeOL ChOI

School of Mathematical Science, Won KWang University,

IK-SAN, 570-749, KOREA.

DAE-HEE Ru

Department of Computer Science, Chung Nam san Up University, HONG-SUNG, 138-240, Korea. 